Bull. Austral. Math. Soc.

VOL. 45 (1992) [343-347]

\title{
ON NORMAL COVERS OF LOCALLY COMPACT SPACES
}

\author{
YUKINOBU YAJIMA
}

Dedicated to Professor Ryosuke Nakagawa on his 60th birthday

In this paper, we deal with the following question: What kind of open covers are normal if they have cushioned open refinements? For this, we prove that an open cover consisting of members with compact closure is a desired one.

\section{INTRODUCTION}

Originally, normal covers of topological spaces were characterised by many forms (for example, see [5, Theorem 1.2]). However, all of these seem to be closely related to local finiteness. So it has raised the following question:

$\left({ }^{*}\right)$ In what kinds of spaces are normal covers characterised by more general properties such as closure-preserving or cushioned ones?

In normal spaces, the author $[7,8]$ characterised normal covers by $\sigma$-cushioned-like properties. Unfortunately, these are not exactly $\sigma$-cushioned property. In this paper, we shall give an answer to the above question. Moreover, we consider when an open cover has an open star-refinement if it has a cushioned open refinement.

Gruenhage [2] showed that a locally compact space $X$ is metacompact if and only if every directed open cover of $X$ has a cushioned refinement. Jiang [3] showed that an orthocompact space $X$ is metacompact if and only if every directed open cover of $X$ has a cushioned refinement. These give a good line for our questions.

Throughout this paper, all spaces are assumed to be Hausdorff.

\section{Definitions}

Let $X$ be a space and $\mathcal{U}$ a cover of $X$. A cover $\mathcal{V}$ of $X$ is a refinement of $\mathcal{U}$ if each member of $\mathcal{V}$ is contained in some member of $\mathcal{U}$. A cover $\mathcal{V}$ of $X$ is a star-refinement (point-star refinement) of $\mathcal{U}$ if for each $V \in \mathcal{V}, \operatorname{st}(V, \mathcal{V})=\cup\left\{V^{\prime} \in \mathcal{V}: V^{\prime} \cap V \neq \emptyset\right\}$ (for each $x \in X, \operatorname{St}(x, \mathcal{V})=\cup\{V \in \mathcal{V}: x \in V\}$ ) is contained in some member of $\mathcal{U}$.

An open cover $\mathcal{U}$ of a space $X$ is said to be normal if there is a sequence $\left\{\mathcal{V}_{n}\right\}$ of open covers of $X$ such that $\mathcal{V}_{0}=\mathcal{U}$ and $\mathcal{V}_{n+1}$ is a star-refinement (or point-star refinement) of $\mathcal{V}_{n}$ for each $n \in \omega$.

Received 30 April 1991

Copyright Clearance Centre, Inc. Serial-fee code: 0004-9729/92 \$A2.00+0.00. 
A subset $U$ of a space $X$ is a cozero set if there is a continuous function $f$ from $X$ into the closed interval $[0,1]$ such that $U=\left\{x \in X: f^{-1}(x)>0\right\}$. It is well-known that an open cover $\mathcal{U}$ of a space $X$ is normal if and only if it has a $\sigma$-locally finite cozero refinement (see [6, Theorem 1.2]).

Let $\mathcal{U}$ and $\mathcal{V}$ be collections of subsets of a space $X$. We say that $\mathcal{V}$ is cushioned in $\mathcal{U}$ [4] if for each $V \in \mathcal{V}$ one can assign a $U_{V} \in \mathcal{U}$ such that for every $\mathcal{V}^{\prime} \subset \mathcal{V}$,

$$
\operatorname{Cl}\left(\cup\left\{V: V \in \mathcal{V}^{\prime}\right\}\right) \subset \cup\left\{U_{V}: V \in \mathcal{V}^{\prime}\right\} .
$$

For two covers $\mathcal{U}$ and $\mathcal{V}$ of a space $X, \mathcal{V}$ is a cushioned refinement of $\mathcal{U}$ if $\mathcal{V}$ is cushioned in $\mathcal{U}$.

\section{RESULTS}

The following is known and the proof is routine. For example, it is found in the proof of (iv) $\rightarrow$ (i) of [1, Theorem 2.6].

Lemma 1. Let $X$ be a space and $\mathcal{U}$ an open cover of $X$. If $\mathcal{U}$ has an open star-refinement, then it has a cushioned open refinement.

Gruenhage [2, Theorem 2] showed:

THEOREM 2. Let $X$ be a locally compact space and $\mathcal{U}$ an open cover of $X$ by sets with compact closure. Then $\mathcal{U}$ has a point-finite open refinement if and only if $\mathcal{U}^{F}$ has a cushioned refinement, where $\mathcal{U}^{F}$ denotes the cover consisting of all finite unions of members of $U$.

We prove the following analogue of Theorem 2. The main idea of the proof of it is due to Gruenhage.

THEOREM 3. Let $X$ be a locally compact space and $\mathcal{U}$ an open cover of $X$ by sets with compact closure. Then $\mathcal{U}$ is normal if and only if it has a cushioned open refinement.

Proof: The "only if" part is obvious from Lemma 1. We show the "if" part.

Let $\mathcal{U}=\left\{U_{\alpha}: \alpha<\kappa\right\}$ such that $\mathrm{Cl} U_{\alpha}$ is compact for each $\alpha<\kappa$, where $\kappa$ is some cardinal. Let $\mathcal{V}$ be a cushioned open refinement of $\mathcal{U}$. We may assume that $\mathcal{V}=$ $\left\{V_{\alpha}: \alpha<\kappa\right\}$ and that $\mathrm{Cl}\left(\bigcup_{\alpha \in \Gamma} V_{\alpha}\right) \subset \bigcup_{\alpha \in \Gamma} U_{\alpha}$ for each $\Gamma \subset[0, \kappa)$ (see [4, Proposition 2.1]). For each $x \in X$, we take an $\alpha(x)<\kappa$ with $x \in V_{\alpha(x)}$.

For each $\alpha<\kappa$, we can construct two collections $\mathcal{U}_{\alpha}$ and $\left\{W(x): x \in S_{\alpha}\right\}$ of open sets in $X$, satisfying the following conditions:

(1) $\mathcal{U}_{\alpha}$ is a countable subcollection of $\mathcal{U}$,

(2) $\left\{U_{\beta}: \beta<\alpha\right\} \subset \bigcup_{\beta \leqslant \alpha} \mathcal{U}_{\beta}$, 
(3) $S_{\alpha}$ is a countable subset of $X \backslash \bigcup_{\beta<\alpha}\left(\cup \mathcal{U}_{\beta}\right)$,

(4) for each $x \in S_{\alpha}, W(x)$ is a cozero set in $X$ such that

$$
x \in W(x) \subset V_{\alpha(x)} \backslash \mathrm{Cl}\left(\cup\left\{V_{\gamma}: U_{\gamma} \in \mathcal{U}_{\beta} \text { and } \beta<\alpha\right\}\right),
$$

(5) $\left(\cup \mathcal{U}_{\alpha}\right) \backslash \bigcup_{\beta<\alpha}\left(\cup \mathcal{U}_{\beta}\right) \subset \cup\left\{W(x): x \in S_{\alpha}\right\}$,

(6) $\left\{U_{\alpha(x)}: x \in S_{\alpha}\right\} \subset \mathcal{U}_{\alpha}$.

This construction is similar to Gruenhage's in the proof of [2, Theorem 2]. The details are left to the reader.

Let $\mathcal{W}=\left\{W(x): x \in S_{\alpha}\right.$ and $\left.\alpha<\kappa\right\}$. It is easily seen from (2), (4) and (5) that $\mathcal{W}$ is a cozero refinement of $\mathcal{U}$. We show that $\mathcal{W}$ is $\sigma$-locally finite in $X$. For each $\alpha<\kappa$, let $G_{\alpha}=\cup\left\{W(x): x \in S_{\alpha}\right\}$. Since each $S_{\alpha}$ is countable, it suffices to show that

$\left.{ }^{*}\right) \quad\left\{G_{\alpha}: \alpha<\kappa\right\}$ is locally finite in $X$.

Assuming the contrary, so say $\left\{G_{\alpha}: \alpha<\kappa\right\}$ is not locally finite at some $p \in X$. Since $U_{\alpha}(p)$ meets infinitely many $G_{\alpha}$ 's, there is a sequence $\left\{\alpha_{n}\right\}$ such that $\alpha_{0}<\alpha_{1}<$ $\cdots<\kappa$ and $U_{\alpha(p)}$ meets all $G_{\alpha_{n}}$ 's. For each $n \in \omega$, pick an $x_{n} \in S_{\alpha_{n}}$ such that $U_{\alpha(p)}$ meets $W\left(x_{n}\right)$. Since $\mathrm{Cl} U_{\alpha(p)}$ is compact, it follows that

$\left(^{* *}\right) \quad\left\{W\left(x_{n}\right): n \in \omega\right\}$ is not locally finite in $X$.

Pick any $x \in \mathrm{Cl}\left(\bigcup_{n \in \omega} W\left(x_{n}\right)\right)$. By (4), we have

$$
x \in \operatorname{Cl}\left(\bigcup_{n \in \omega} V_{\alpha\left(x_{n}\right)}\right) \subset \bigcup_{n \in \omega} U_{\alpha\left(x_{n}\right)} .
$$

Take a $k \in \omega$ with $x \in U_{\alpha\left(x_{k}\right)}$. Since $\bigcup_{\alpha<\kappa} \mathcal{U}_{\alpha}$ covers $X$, take some $\delta<\kappa$ such that $x \in\left(\cup \mathcal{U}_{\delta}\right) \backslash \bigcup_{\beta<\delta}\left(\cup \mathcal{U}_{\beta}\right)$. By (6), we have $U_{\alpha\left(x_{k}\right)} \in \mathcal{U}_{\alpha_{k}}$. So we get $\delta \leqslant \alpha_{k}$. By (5), take some $y \in S_{6}$ with $x \in W(y)$. It follows from (4) and (6) that

$$
x \in W(y) \subset V_{\alpha(y)} \subset U_{\alpha(y)} \in \mathcal{U}_{\delta} .
$$

Take any $n>k$. Since $\alpha_{n}>\alpha_{k} \geqslant \delta$, it follows from (4) that

$$
W\left(x_{n}\right) \cap V_{\alpha(y)} \subset W\left(x_{n}\right) \cap\left(\cup\left\{V_{\gamma}: U_{\gamma} \in \mathcal{U}_{\beta} \text { and } \beta<\alpha_{n}\right\}\right)=\emptyset .
$$

Hence $x \notin \mathrm{Cl}\left(\cup\left\{W\left(x_{n}\right): n>k\right\}\right)$. This contradicts $\left(^{* *}\right)$, so that we have shown $\left({ }^{*}\right)$. Thus $\mathcal{W}$ is a $\sigma$-locally finite cozero refinement of $\mathcal{U}$, and so $\mathcal{U}$ is normal.

Question A: In Theorem 3, can the "cushioned" be replaced by " $\sigma$-cushioned"? 
An open cover $\mathcal{U}$ of a space $X$ is interior-preserving if $\mathrm{nU}^{\prime}$ is open in $X$ for each $\mathcal{U}^{\prime} \subset \mathcal{U}$. A space $X$ is said to be orthocompact if every open cover of $X$ has an interior-preserving open refinement.

Jiang [3, Theorem 2.1] showed

TheOREM 4. Let $X$ be a space and $\mathcal{U}$ an interior-preserving open cover of $X$. Then $\mathcal{U}$ has an open point-star refinement if and only if it has a cushioned refinement.

We can get an analogue of Theorem 4.

Theorem 5. Let $X$ be a space and $\mathcal{U}$ an interior-preserving open cover of $X$. Then $\mathcal{U}$ has an open star-refinement if and only if it has a cushioned open refinement.

ProOF: The "only if" part follows immediately from Lemma 1.

Let $\mathcal{V}$ be a cushioned open refinement of $\mathcal{U}$. Let $\mathcal{U}=\left\{U_{\alpha}: \alpha \in A\right\}$. We may assume that $\mathcal{V}=\left\{V_{\alpha}: \alpha \in A\right\}$ and that $\mathrm{Cl}\left(\bigcup_{\alpha \in B} V_{\alpha}\right) \subset \bigcup_{\alpha \in B} U_{\alpha}$ for each $B \subset A$.

For each $x \in X$, choose an $\alpha(x) \in A$ with $x \in V_{\alpha(x)}$, and let

$$
W(x)=\left(\cap\left\{U_{\alpha}: x \in U_{\alpha}\right\} \cap V_{\alpha(x)}\right) \backslash \operatorname{Cl}\left(\cup\left\{V_{\alpha}: x \notin U_{\alpha}\right\}\right)
$$

Let $\mathcal{W}=\{W(x): x \in X\}$. Since each $W(x)$ is an open neighbourhood of $x$ in $X, \mathcal{W}$ is an open cover of $X$. Pick any $x \in X$. It suffices to show that $\operatorname{St}(W(x), \mathcal{W}) \subset U_{\alpha(x)}$. Suppose that $W(x) \cap W(y) \neq \emptyset$. If $y \notin U_{\alpha(x)}$, we have $W(y) \cap V_{\alpha(x)}=\emptyset$. Since $W(x) \subset V_{\alpha(x)}$, this is a contradiction. So $y \in U_{\alpha(x)} \in \mathcal{U}$. Therefore it follows that $W(y) \subset \cap\left\{U_{\alpha}: y \in U_{\alpha}\right\} \subset U_{\alpha(x)}$.

QUESTION B: Let $X$ be an orthocompact space and $\mathcal{U}$ an open cover of $X$. If $\mathcal{U}$ has a cushioned open refinement, does it have an open star-refinement?

\section{REFERENCES}

[1] D.K. Burke, 'Covering properties', in Handbook of Set Theoretic Topology, Edited by K. Kunen and J.E. Vaughan, pp. 347-422 (North-Holland, Amsterdam, 1984).

[2] G. Gruenhage, 'Games, covering properties and Eberlein compacts', Topology Appl. 23 (1986), 291-297.

[3] S. Jiang, 'On a Junnila's problem', $Q$ \& $A$ in General topology 6 (1988), 43-47.

[4] E. Michael, 'Yet another note on paracompact spaces', Proc. Amer. Math. Soc. 10 (1959), 309-314.

[5] K. Morita, 'Paracompactness and product spaces', Fund. Math. 50 (1962), 223-236.

[6] K. Morita, 'Products of normal spaces with metric spaces', Ann. of Math. 154 (1964), 365-382.

[7] Y. Yajima, 'A characterization of normal covers of a normal space', Glas. Mate. 18 (1983), 331-334. 
[5]

[8] Y. Yajima, 'A characterization of normal covers of a normal space II', Glas. Mate. 24 (1989), 401-403.

Department of Mathematics

Kanagawa University

3-27-1 Rokkakubashi

Yokahama 221

Japan 